\title{
Cilia, asymmetry, and genetic disease
}

The length and movement of motile cilia - microscopic hairlike organelles on the outside of our cells - have a remarkable effect on the asymmetric development of embryos,
allowing organs to grow in the correct places in our bodies. Dr Susana Lopes and her
team at the LYSOCIL project are investigating rare genetic diseases affecting the cilia, and how these can be modelled using zebrafish to better understand them, bringing us closer to potential treatments. The team have showed that levels of the Polycys in 2 protein, modulated in cilia, are critical for the normal development of an embryo.

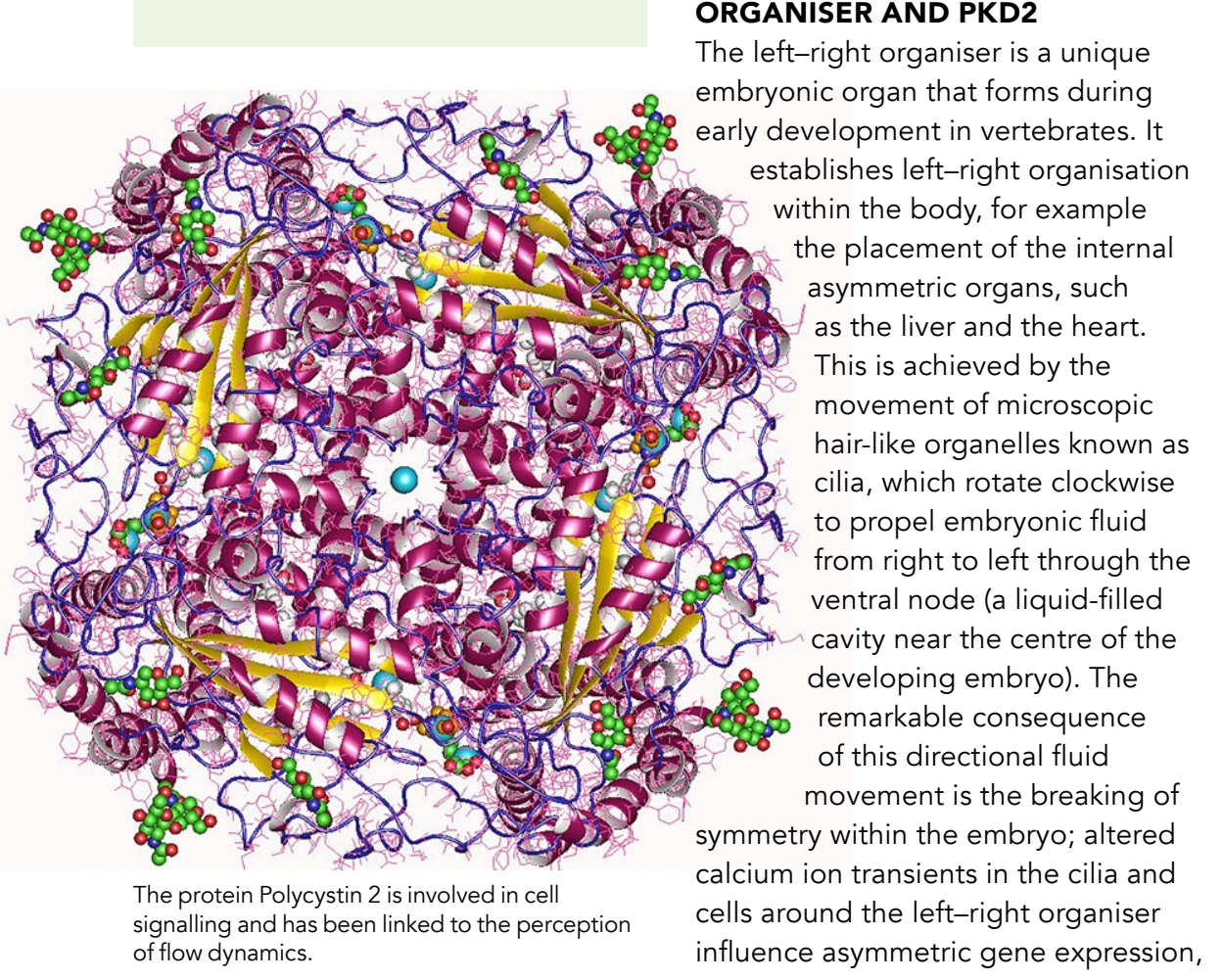

eft-right asymmetry is a phenomenon widely observed in nature. While many animals may appear symmetrical, asymmetry is pervasive in every major subset of the and can be as subtle as the colling direction of a snail's sh of fiddent as the size difference - the asymmetrical property of an object which is non-superimposable biomolecules such as amino acids and sugars, as well as the majority of cells. However, the mechanisms that oscopic asymmetry in an organism's tissues or entire body, starting from a symmetrical bundle of embryonic cells, are complex and not fully understood.

\section{THE LEFT-RIGHT} The aim of the LYSOCIL Project, led by researchers at NOVA Medical School, of the underlying causes of diseases affecting the cilia, known as

ciliopathies. Many of these diseases are very rare, for example primary ciliary dyskinesia (affecting around 1 in 10,000 people) and autosomal dominant polycystic kidney disease (affecting around 1 in 1,000 people), but they are debilitating condition causing abnormal function of the affected areas.

The team have previously demonstrated the use of zebrafish left-right organisers - known as Kupffer's vesicles (KVs) in fish - as an effective model organ to study renal cysts associated with autosoma dominant polycystic kidney disease.
They concluded that a reduced level of

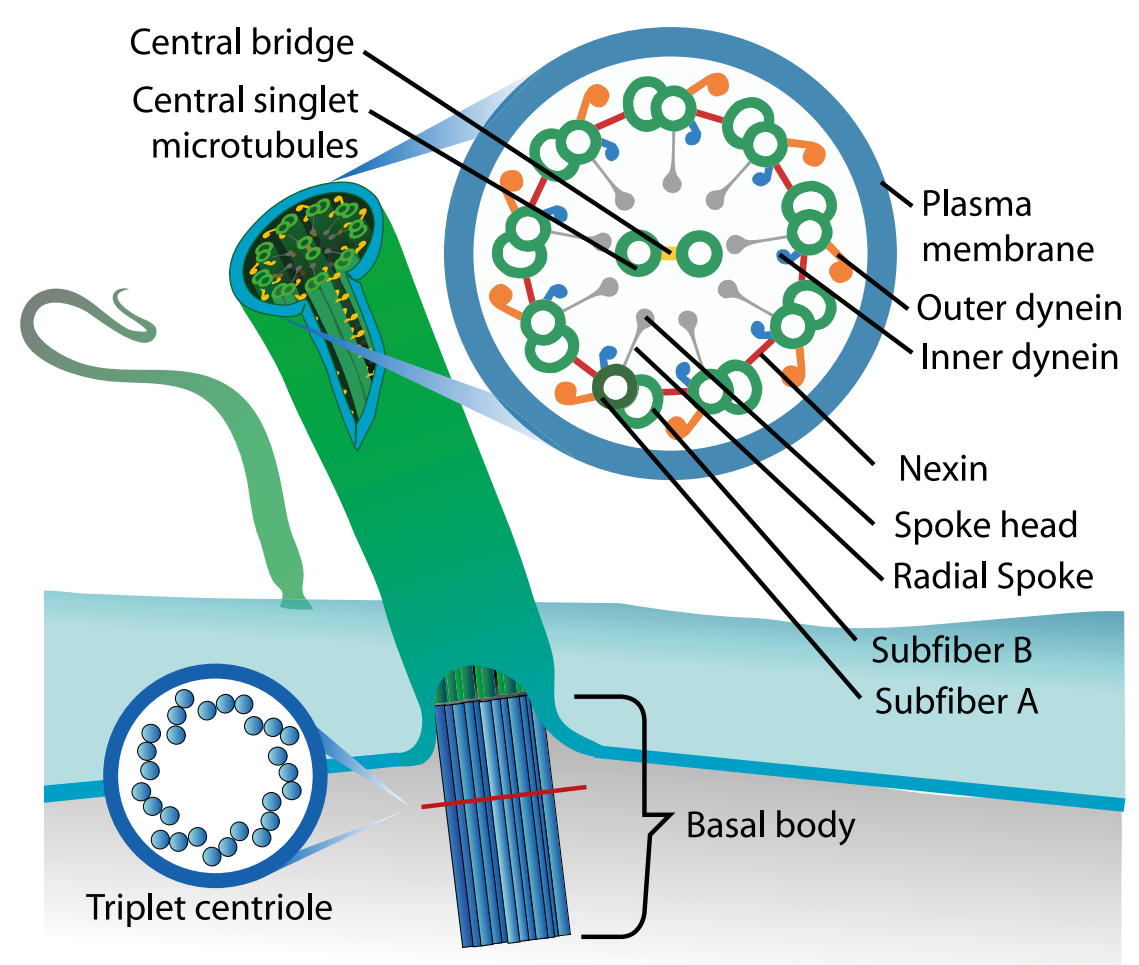

The mechanisms that produce macroscopic asymmetry in an organism are complex and not fully understood.

Pkd2 was associated with overinflation of Using antisense technology - a the model cyst caused by increased fluid method of targeting genes to stop secretion into the $\mathrm{KV}$; this highlighted them producing certain proteins Pkd2 as an important regulator of the pkd2 gene was knocked down healthy kidney function and reduced to produce embryonic KV cells with levels of the protein as a potential causal decreased levels of Pkd2 protein factor for cyst inflation. Nevertheless, while the rest of the embryo had the exact mechanism by which Pkd2 normal levels of Pkd2. The researchers these cilited cells (ind ding the ones to compare the flow speed of in leftright organisers and renal cysts) remained unclear.

\section{POLYCYSTIN 2 AND}

\section{EMBRYONIC CILIA}

A particular goal for Dr Lopes and her collaborators at the LYSOCIL Project. was to reveal the precise activity of Pkd2 levels and their effect on the zebrafish KV. In doing so, they could establish how this protein exerts its effects on left-right development and flow dynamics, shedding light on how rare diseases might be caused.

Initially, the team investigated the $\mathrm{KV}$ flow characteristics in zebrafish embryonic fluid (inside the left-right organiser) between these knocked wild type (unchanged) embryos that had a normal concentration of Pkd2. The researchers found that the flow speed in the Pkd2-deficient embryos averaged just half that of the wild type embryos, with a rate of 5 ums-1 compared to $10 \mu \mathrm{ms}-1$. This is likely attributable to the cilia beat frequency, which was also found to be lower in the pkd2 knockdown cells. The team also found a significant decrease in cell height in the affected embryos, highlighting abnormal development of the $K V$ cells.

Dr Lopes and her colleagues next investigated the resulting length of cilia grown by the $\mathrm{KV}$ cells. Compare was shorter on average in the $p k d 2$ knockdown embryos, with a significant decrease compared to the wild types. Interestingly, subsequent injection of $p k d 2 \mathrm{mRNA}$ into the deficient embryonic cells caused the cilia length to increase and generated a partial rescue, though not to the level seen in the wild type cells.

The researchers wanted to take a closer look at these previously unreported findings on cilia length, to understand their implications for ciliopathic diseases. They first injected the embryos with arl13b-GF mRNA which makes all cilia become luorescently green, and examined cilia of the Pkd2-deficient embryos,

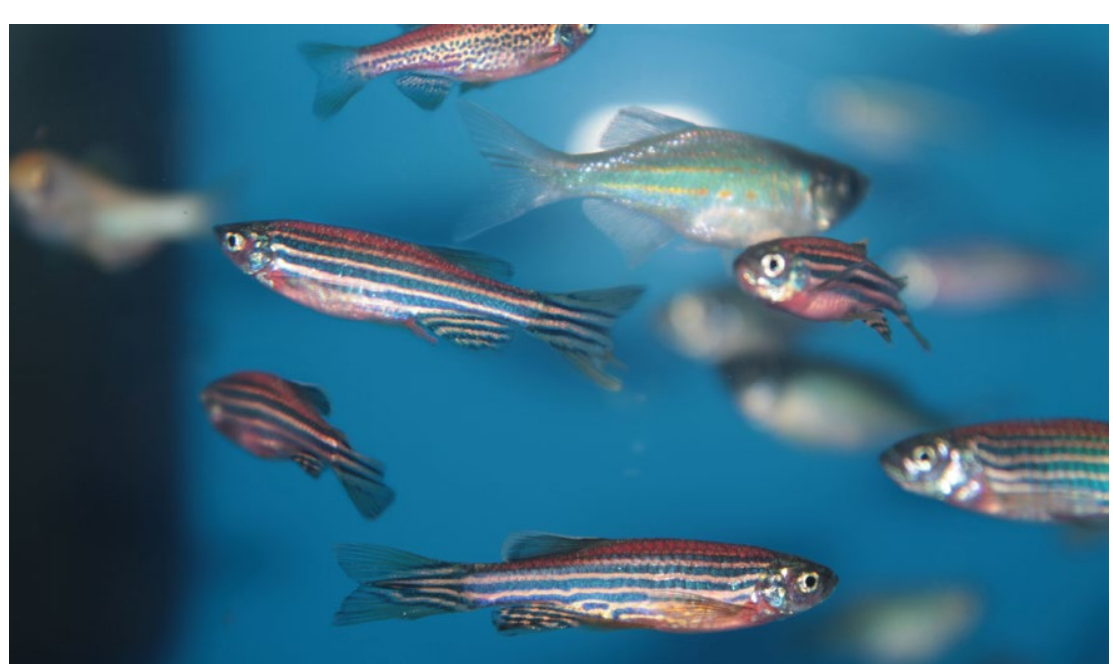

Dr Lopes' work on Zebra fish helps us understand ciliopathies in humans. 

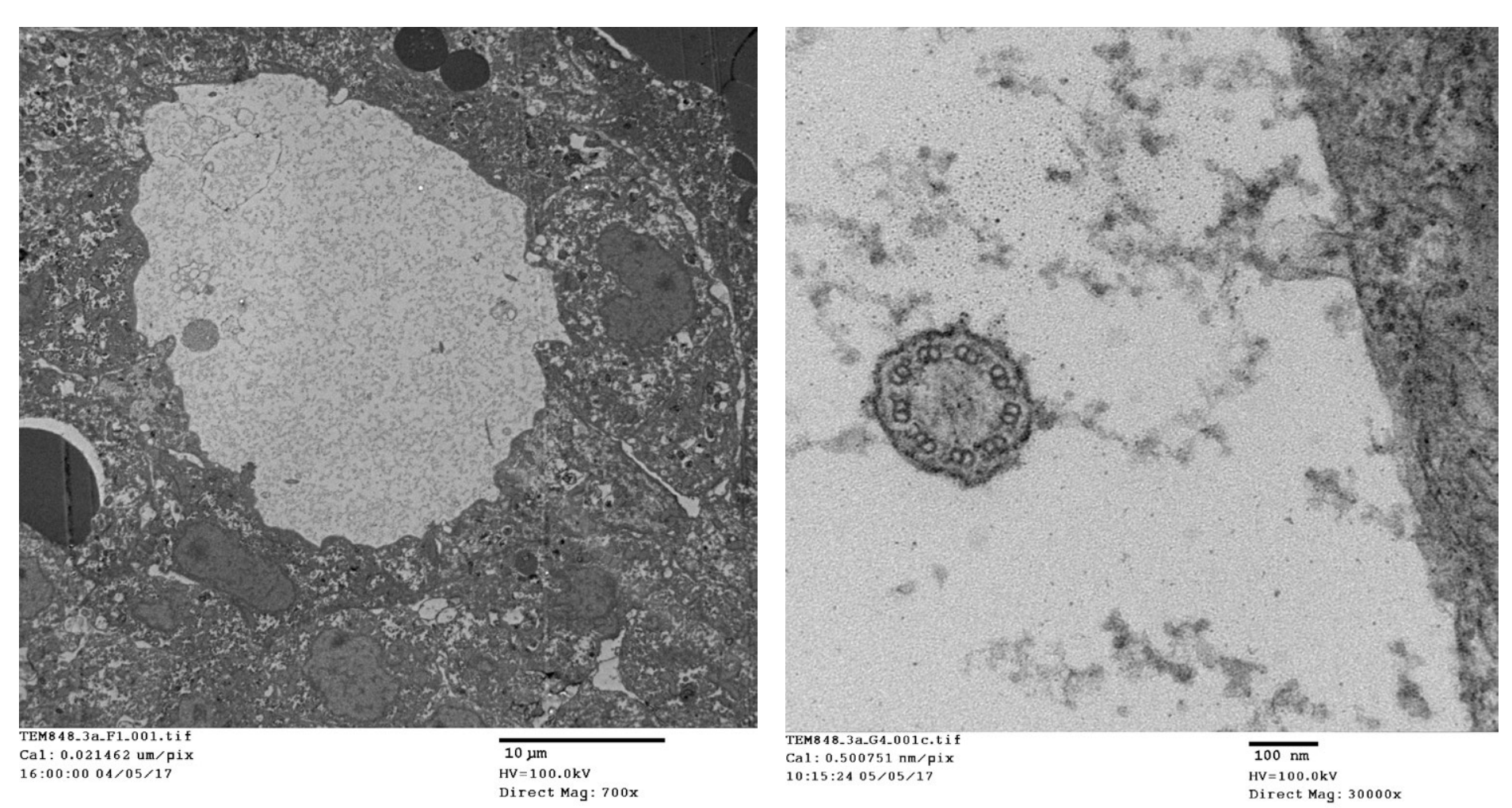

Transmission electron microscopy photographs. Left: With low amplification to show a cross section through the lumen of the Kupffer's vesicle, the zebrafish
left-right organiser. Right: With large amplification to show a crosss section through one motile cilia from one Kupffer's vesicle cell.

in which there was a reduction of more dand5, which in zebrafish is expressed on opposite sides. In nature, these two than $10 \%$ in the number of motile vs mostly on the right side of the KV. In the conditions are both extremely rare, $\begin{array}{ll}\text { immotile cilia. Next, } & \text { affecting only } \\ \text { they remeasured } & \text { an 10,000 }\end{array}$

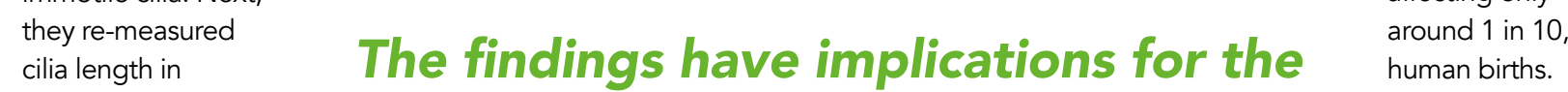
live embryos,
between Pkd2- further understanding of rare ciliopathies, Overall, the deficient mutated including autosomal dominant researchers' results clearly demonstrate nonmutated KVs. polycystic kidney disease and primary that reduced The mean cilia length was found

the Pkd2-deficient cells, with an average 8.02 micrometres in the wild type cells expressing Pkd2 normally The resulting decrease in flow speed is dramatic - it has been previously reported that the flow produced by a cilium is proportiona to the cube of its length, so the observed decrease is expected to reduce the rate of flow by more than hal.

\section{ALTERED SYMMETRY AND} ORGAN POSITIONIN The researchers finally looked to investigate the effects of reduced Pkd2 levels on the symmetry of gene expression and the resulting positioning of organs in the zebrafish. The first gene known to be expressed asymmetrically
during the embryo's development is cilliary dyskinesia.

Pkd2-deficient embryos created using antisense technology, the resulting Pkd2 with only $22 \%$ asymmetric distribution on the right side compared to $84 \%$ in the wild type.

When the zebrafish embryos had developed further, the team examined their organ laterality. In the wild types, $97.5 \%$ of fish displayed situs solitus - the normal positioning of the heart and liver on the left side of the body. In the modified group, this normal organ fish, $21 \%$ exhibited situs inversus, in which the internal organs develop in the opposite orientation (heart and liver both on the right side), and $21 \%$ were positioning fell to $54 \%$. Of the remaining embryonic frequently Consequently, the flow rate of embryonic fluid decreases dramatically leading to left-right defects during growth. The findings have implication for the further understanding of rare and debilitating ciliopathies, including autosomal dominant polycystic kidney disease, as Dr Lopes and her colleagues have demonstrated that the cyst inflation question can be modelled effectively using zebrafish KVs. It also highlights questions in this field that need to be studied further - for example, are shorter cilia the cause or the effect of abnormallyshaped cells? Additional research in this area will continue to shed light on the factors behind such diseases, helping us lead to abnormal

cexhibited in misshapen

Dr Susana Lopes

E: susana.lopes@nms.unl.pt T: +351218803101

Research Objectives

Dr Lopes studies cilia length and motility regulation in zebra fish in order to understand ciliopathies in humans.

\section{Detail}

CEDOC

OVA Medical School

CEDC III Rua Câmara Pestana nº 6, 6-A I 1150082 Lisboa I Portugal

Bio

Susana Lopes is the group leader of the Cilia Regulation and Disease Laboratory at CEDOC - Nova Medica School (NMS). She is an invited professor at NMS and has a total of 34 peer-reviewed publications. Lopes' lab carries out fundamental research on motile cilia and runs a patient diagnostic service for Primary Ciliary Dyskinesia.

Funding

This aricle was supported by the LYSOCIL project 2020 research and innovation under grant agreement No 811087.

\section{Collaborator}

Thom L Saúde; Thomas JohnsonIGC Image a Did Faclities; and to Martin Blum, Pera Pennekamp, Rebecca Burdine and Dominic Norris for discussions over the years.

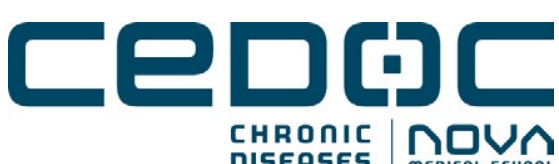

$\mathbf{N} \bigcirc \mathbf{V \Lambda}$ SCHOOLL

\section{References}

Jacinto R., Sampaio P., et al. (2021). Pkd2 Affects Cilia Length and Impacts LR Flow Dynamics and Dand5. Front Cell Dev Biol., 9 (624531), 1-10. doi.org/10.3389/fcell.2021.624531

Roxo-Rosa M., et al. (2015). The zebrafish Kupffer's vesicle as a model system for the molecular mechanisms by which the 4, 1356-1366. doi.org/10.1242/bio.014076

Yoshiba S., et al. (2012). Cilia at the node of mouse embryos sense fluid flow for left-right determination via science. 1222538

\section{Personal Response}

Your team is looking to develop a greater understanding of ciliopathies so that potential is the next major research question that needs to addressed to make this possible?

II Directly related to the Pkd2 ion channel topic, right embryonic organ and the kidneys. It is crucial to understand the role of Pkd2 in motile cilia, in nonmotile cilia and in cell membranes for the laterality that stem from the left-right embryonic organises. For the kidney cystic disease (ADPKD) it is important to discern the Pkd2 role in the primary cilia (non-motile present in the kidney tubules and cysts) and in the basolateral plasma membrane as well as in the ER

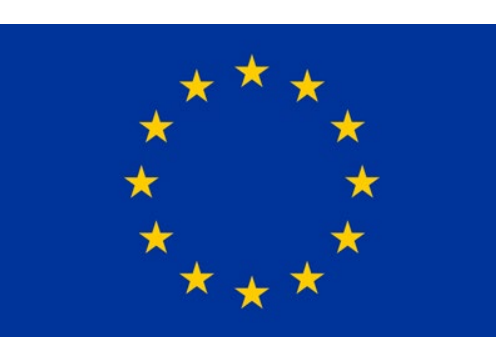

